\title{
Effects of ultrasound on estradiol level, bone mineral density, bone biomechanics and matrix metalloproteinase-13 expression in ovariectomized rabbits
}

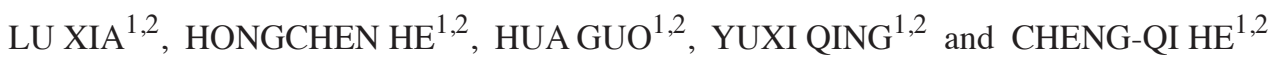 \\ ${ }^{1}$ Department of Rehabilitation, West China Hospital, Sichuan University; \\ ${ }^{2}$ Rehabilitation Key Laboratory of Sichuan, Chengdu, Sichuan 610041, P.R. China
}

Received December 12, 2014; Accepted July 2, 2015

DOI: $10.3892 / \mathrm{etm} .2015 .2673$

\begin{abstract}
The aim of the present study was to observe the effect of ultrasound (US) on estradiol level, bone mineral density (BMD), bone biomechanics and matrix metalloproteinase-13 (MMP-13) expression in ovariectomized (OVX) rabbits. A total of 28 virgin New Zealand white rabbits were randomly assigned into the following groups: Control (control group), ovariectomy (OVX group), ovariectomy with ultrasound therapy (US group) and ovariectomy with estrogen replacement therapy group (ERT group). At 8 weeks after ovariectomy, the US group received ultrasound treatment while the ERT group were orally treated with conjugated estrogens, and the control and OVX groups remained untreated. The estradiol level, BMD and bone biomechanics, cartilage histology and the MMP-13 expression were analyzed after the intervention. The results indicate that the US treatment increased estradiol level, BMD and bone biomechanical function. Furthermore, the US treatment appeared to improve the recovery of cartilage morphology and decreased the expression of MMP-13 in OVX models. Furthermore, the results suggest that 10 days of US therapy was sufficient to prevent the reduction of estradiol, BMD and bone biomechanical function, to protect osteoarthritis cartilage structure, and to reduce MMP-13 transcription and expression in OVX rabbits. Therefore, US treatment may be a potential treatment for postmenopausal osteoarthritis and osteoporosis.
\end{abstract}

\section{Introduction}

Osteoarthritis (OA) is a disease characterized by degradation of articular cartilage, osteophyte formation, subchondral

Correspondence to: Professor Cheng-Qi He, Department of Rehabilitation, West China Hospital, Sichuan University, 37 Guoxue Xiang Street, Chengdu, Sichuan 610041, P.R. China

E-mail: hechqi@gmail.com

Key words: ultrasound therapy, estradiol, osteoporosis, osteoarthritis, matrix metalloproteinase-13 sclerosis and synovial proliferation, which results in the loss of joint function and disability (1). The loss of articular cartilage matrix has been shown to involve the cleavage of native collagen by matrix metalloproteinases (MMPs) (2). MMP-13, also known as collagenase 3 , is crucially involved in OA as it preferentially degrades type II collagen (3). In previous studies, the expression of MMP-13 was observed to be markedly increased in patients with OA $(2,4)$. Osteoporosis (OP) is a disorder characterized by reduced bone mass, leading to diminished physical bone strength and increased susceptibility to fracture (5). OA and OP are the most common musculoskeletal disorders among elderly populations, and are associated with estrogen deficiency (6-8). Furthermore, previous studies have demonstrated that ovariectomy may be used as an experimental intervention for treating postmenopausal OA and OP (9-12).

Estrogen replacement therapy (ERT) is an effectiveness treatment for the prevention of OA and OP (13). A number of large-scale epidemiological studies have demonstrated a decreased incidence of OA- and OP-induced hip fracture in patients who were administered estrogen compared with untreated patients; however, long-term ERT is associated with an increased risk of breast and uterine cancers, in addition to cardiovascular and thromboembolic disease $(14,15)$. In response to these side-effects, studies have been conducted with the aim of identifying alternative anti-osteoarthritic and anti-osteoporotic approaches with comparable effectiveness to estrogen.

Ultrasound (US) therapy is based on the application of high frequency sound waves to the tissues of the body to induce mechanical or thermal effects (16). In in vivo studies, US has been demonstrated to improve mechanical strength and bone mineral density (BMD) $(17,18)$ and to repair joint cartilage in animal models of cartilage injury (19). In in vitro studies, US therapy has been shown to effectively suppress osteoclast formation (20), enhance osteoblast formation and function (21), stimulate the synthesis of extracellular matrix (ECM) proteins in cartilage, and alter chondrocyte maturation and endochondral bone formation $(22,23)$. A systematic review of clinical studies indicated that US is efficacious for decreasing pain and may improve physical function in patients with knee OA (24). However, contrary results have been also 
reported (25). Although a number of studies have indicated the utility of ultrasound and estrogen in the treatment of OA and OP (14-19), it is not clear which therapeutic strategy is more effective in improving serum estrogen levels, BMD, bone biomechanical strength, cartilage structure and MMP-13 expression.

The aim of the present study was to investigate the efficacy of US and ERT in the prevention and treatment of ovariectomized (OVX) rabbits. Efficacy was assessed by evaluating a number of parameters, including serum estrogen level, BMD, bone biomechanical function and cartilage structure, in addition to MMP-13 transcription and translation. In addition, the present study aimed to determine whether US treatment induces equivalent therapeutic effects compared with estrogen treatment for postmenopausal OA and OP in OVX rabbits.

\section{Materials and methods}

Laboratory animals. A total of 28 virgin, 5-month-old, New Zealand white (NZW) rabbits, weighing 2.5-3 kg, were purchased from the Experimental Animal Center of West China Hospital, Sichuan University (SYXK-Sichuan-2009-045; Sichuan, China). The rabbits were allocated at random into four groups ( $n=7$ per group): Normal control with no treatment (control group); ovariectomized control (OVX group); ovariectomy with ultrasound therapy (US group); and ovariectomy with exogenous estrogen replacement therapy (ERT group). A total of 7 rabbits from each group were tested in the BMD, bone biomechanics, histological analysis, polymerase chain reaction $(\mathrm{PCR})$ and western blot analysis.

Animals were housed in individual cages with a 12:12-h light-dark cycle at $20-26^{\circ} \mathrm{C}$, and received a standard laboratory diet and drinking water. All rabbits were subjected to the bilateral ovariectomy, with the exception of the control group. All animal care and experimental procedure were approved by the Institutional Animal Care Center of Sichuan University and Ethics Committee on Research Animal of People's Republic of China.

Bilateral ovariectomy. Bilateral ovariectomy was performed by anesthetizing rabbits with $5 \%$ chloral hydrate $(3 \mathrm{ml} / \mathrm{kg})$ and placing the animal in a supine position on a rabbit fixation board (Hepu Co.Ltd, Tianjin, China) as previously described (26). The abdominal region of the anesthetized rabbit was aseptically prepared and a $5-\mathrm{cm}$ incision was made on the middle abdomen of the rabbit. The ovarian artery and vein were ligated and the bilateral ovaries were then excised. Following irrigation with sterile saline solution, the wounds were closed in layers. Rabbits received appropriate postoperative care and were allowed freedom of activity within their individual cages.

Treatment. US therapy was performed using a commercial, clinically-approved proprietary SonicMaster ES-2 ultrasound device (Ultrasonic apparatus ES-2, OG Giken Co., Ltd., Okayama, Japan). At 8 weeks after the ovariectomy, US with an intensity of $300 \mathrm{~mW} / \mathrm{cm}^{2}$ and a frequency of $1 \mathrm{MHz}$ was administered to the US group for $10 \mathrm{~min} /$ day over 10 days (27). Bilateral legs were shaved and the rabbits were fixed on a rabbit fixation board in a supine position. US transducers were attached to the medial aspect of the femur and knee, with a moving speed of $1 \mathrm{~cm} / \mathrm{sec}$ manually. Standard ultrasonic coupling gel was applied between the transducer head and the limb to minimize reflection and attenuation of the US energy. Concurrently, rabbits in the ERT group were orally treated with conjugated estrogens tablets (Pfizer, New York, USA) at $0.625 \mathrm{mg} / \mathrm{kg} /$ day for 10 days $(28,29)$. The OVX and control groups remained untreated. All rabbits were anesthetized by $5 \%$ chloral hydrate $(3 \mathrm{ml} / \mathrm{kg})$ and sacrificed at week 10 by air embolism.

Serum estradiol assay. Blood samples were collected from each experimental animal at baseline, week 8 and week 10 to assess the serum levels of $17 \beta$-estradiol. A $~ 2-\mathrm{ml}$ serum sample was extracted from each blood sample by centrifugation at $2,000 \mathrm{x} \mathrm{g}$ for $20 \mathrm{~min}$, and stored at $-80^{\circ} \mathrm{C}$ until required for analysis. Estradiol levels were detected using an enzyme-linked immunosorbent assay (ELISA), following the manufacturer's instructions (R\&D Systems, Inc., Minneapolis, MN, USA).

$B M D$ testing. The femur from the rabbit was separated and stored at $-20^{\circ} \mathrm{C}$ for $\mathrm{BMD}$ and biomechanical testing. The average BMD of the entire femur was detected with dual-energy X-ray absorptiometry (DEXA) using a GE Lunar iDXA Bone Densitometer (GE Healthcare Life Sciences, Fairfield, CT, USA). An individual femur was immersed in 2-cm deep deionized water, which accurately simulated the bone surrounding with soft tissues observed in the clinical setting (30). The neck and head of the femur was oriented perpendicularly to the DEXA beams, which limited the rotational effects that can alter DEXA measurements (31). Each femur was positioned at the same place to ensure consistency of results in the scanning process. The data were collected and analyzed by the small animal research software (Lunar iDAXTM, version 13.6, GE provided by GE Healthcare Life Sciences (Madison, WI, USA).

Bone biomechanics. Bone strength was determined by three-point bending testing of the femur, using an Instron 8874 axial-torsion fatigue testing system (Instron Co., Boston, TH, USA), which included a three-point bending apparatus. Femurs were placed on supports and were bent until the $40 \%$ maximum load was reached by lowering a centrally placed blade (1-mm width) at a constant crosshead speed of $10 \mathrm{~mm} / \mathrm{min}$. Maximum load (N) was for each femur was recorded by a computer interfaced with the Instron analyzer and using WaveMatrixTM, Version V6.7, Instron Co. Ltd (Boston, TH, USA) software.

Histological analysis. The distal femur of the rabbit knee was separated and fixed in 10\% buffered formalin. Following decalcification, the samples were sequentially dehydrated in alcohol and embedded in paraffin blocks. The samples were sectioned at a thickness of $5 \mu \mathrm{m}$ for histopathological classification. Sections were stained with hematoxylin and eosin (H\&E). Modified Mankin scoring (32) was conducted to grade the histopathological classification of articular cartilage for OA (Table I). Scoring was performed by two independent 
Table I. Modified Mankin scoring system.

\begin{tabular}{lc}
\hline Category & Score \\
\hline Structure & \\
Normal & 0 \\
Surface irregularities & 1 \\
Pannus and surface irregularities & 2 \\
Clefts to transitional zone & 3 \\
Clefts to radial zone & 4 \\
Clefts to calcified zone & 5 \\
Complete disorganization & 6 \\
Cells & \\
Normal & 0 \\
Diffuse hypercellularity & 1 \\
Cloning & 2 \\
Hypocellularity & 3 \\
Safranin O staining & \\
Normal & \\
Slight reduction & 0 \\
Moderate reduction & 14 \\
Severe reduction & 1 \\
No dye noted & 2 \\
Tidemark integrity & 2 \\
Crossed by blood vessels & 3 \\
Minimal score & 4 \\
Maximal & \\
\hline & \\
\hline
\end{tabular}

observers, blind to the origin of the sections. Digital images were captured using an optical microscope (Eclipse 80i, Nikon, Tokyo, Japan) at a magnification of x40.

RNA extraction and reverse transcription-quantitative polymerase chain reaction ( $R T-q P C R)$. Cartilage tissue was obtained from the femoral condyle and the tibial plateau of each rabbit and immediately stored in liquid nitrogen for RT-qPCR analysis and western blot analysis. Total RNA was isolated from the cartilage using TRIzol reagent (Takara Holdings Inc., Kyoto, Japan). A $0.5-\mu$ g total RNA sample from each specimen was used for cDNA synthesis and RNA amplification using one-step RT-qPCR, following the instruction of PrimeScript One Step RT-PCR kit (Takara Holdings Inc.). The PCR procedure was programmed to proceed as follows: $50^{\circ} \mathrm{C}, 30 \mathrm{~min}, 94^{\circ} \mathrm{C}, 2 \mathrm{~min}, 1$ cycle; $94^{\circ} \mathrm{C}, 30 \mathrm{sec}$, $54^{\circ} \mathrm{C}$ [melting temperature (Tm) of MMP-13] $/ 49^{\circ} \mathrm{C}(\mathrm{Tm}$ of GAPDH), $30 \mathrm{sec}, 72^{\circ} \mathrm{C}, 30 \mathrm{sec}, 30$ cycles; $72^{\circ} \mathrm{C}, 5 \mathrm{~min}$, 1 cycle; and $16^{\circ} \mathrm{C}, 20 \mathrm{~min}$.

Each experiment was repeated at least three times. The sequences of reaction products were confirmed by agarose gel electrophoresis, and the negative control reactions run in the absence of reverse transcription did not generate any products. The mRNA expression levels of MMP-13 were determined relative to those of GAPDH. The specific sequences of the

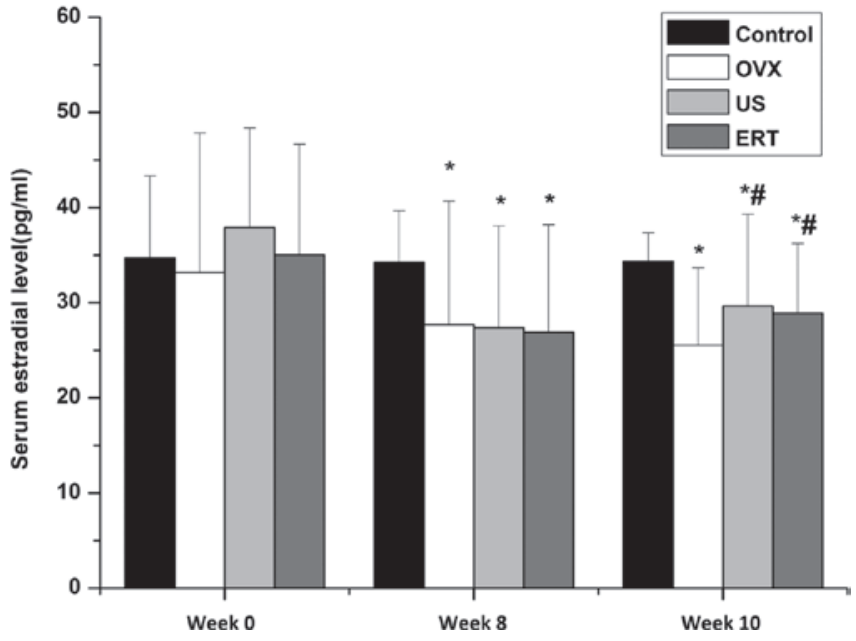

Figure 1. Serum estradiol level in the control, OVX, US and ERT groups. ${ }^{*} \mathrm{P}<0.05$ vs. control group; ${ }^{\#} \mathrm{P}<0.05$ vs. OVX group at week 10 . No significant difference among the four groups at week 0 . Values are expressed as the mean \pm standard deviation $(n=7)$. OVX, ovariectomized; US, ultrasound; ERT, estrogen replacement therapy.

PCR primers for MMP-13 and GAPDH and the expected PCR product lengths are listed in Table II.

Western blot analysis. The cartilage was lysed at $4^{\circ} \mathrm{C}$ in radioimmunoprecipitation assay buffer containing protease inhibitor cocktail, $1 \mathrm{mM}$ 4-(2-aminoethyl)benzenesulfonyl fluoride and $2 \mathrm{mM}$ dithiothreitol. The lysate concentration was measured in duplicate using the Bradford assay (Bio-Rad Laboratories, Inc., Hercules, CA, USA). Samples were separated by SDS-PAGE and electro-transferred to PVDF membranes. Non-specific binding was blocked by $5 \%$ non-fat milk in Tris-buffered saline containing $0.05 \%$ Tween 20 for $1 \mathrm{~h}$. Membranes were incubated with primary antibodies (rabbit monoclonal anti-MMP-13, 1:1,000, Wuhan Boster Biological Technology, Ltd., Wuhan, China; rabbit monoclonal anti-GAPDH, 1:1,000, Santa Cruz Biotechnology, Inc., Santa Cruz, CA, USA) at $4^{\circ} \mathrm{C}$ overnight to detect the specific protein expression. Membranes were subsequently incubated with the anti-rabbit horseradish peroxidase (HRP)-conjugated secondary antibody (Wuhan Boster Biological Technology, Ltd.) in 5\% blocking buffer for $1 \mathrm{~h}$ at room temperature. Next, streptavidin-HRP was applied in 5\% blocking buffer for 30-60 min at room temperature, followed by incubation with BeyoECL Plus reagents A and B (BeyoECL Plus, Beyotime Institute of Biotechnology, Shanghai, China) for 2 min prior to visualization using an HRP-driven chemiluminescence reaction (Beyotime Institute of Biotechnology) and exposure to autoradiographic film in the dark room. The expression levels of MMP-13 were normalized against GAPDH.

Statistical analysis. Results are expressed as the mean \pm standard deviation. Statistical analyses were conducted using SPSS, version 20.0 (IBM SPSS, Armonk, NY, USA). One-way analysis of variance followed by least significant difference correlation and $\chi^{2}$ test were used to compare differences among all groups. The Kruskal-Wallis test was used if the homogeneity of the variance test showed heterogeneity. $\mathrm{P}<0.05$ was considered to indicate a statistically significant difference. 
Table II. Primer sequences and PCR product lengths.

\begin{tabular}{llcc}
\hline Primer & \multicolumn{1}{c}{ Sequence 5'-3' direction } & NCBI Accession & PCR product length (bp) \\
\hline MMP-13 & F GTTATCTGAACTGGATT & AF059201 & 147 \\
GAPDH & R TCTAGGGTAGTCTTGGTC & & 226 \\
& F GGTCGGAGTGAACGGATTT & NM_001082253
\end{tabular}

All accession numbers refer to those on the NCBI database, and are sequenced from rabbits. PCR, polmerase chain reaction; NCBI, National Center for Biotechnology Information (US); MMP-13, matrix metalloproteinase-13; GAPDH, glyceraldehyde 3-phosphate dehydrogenase.

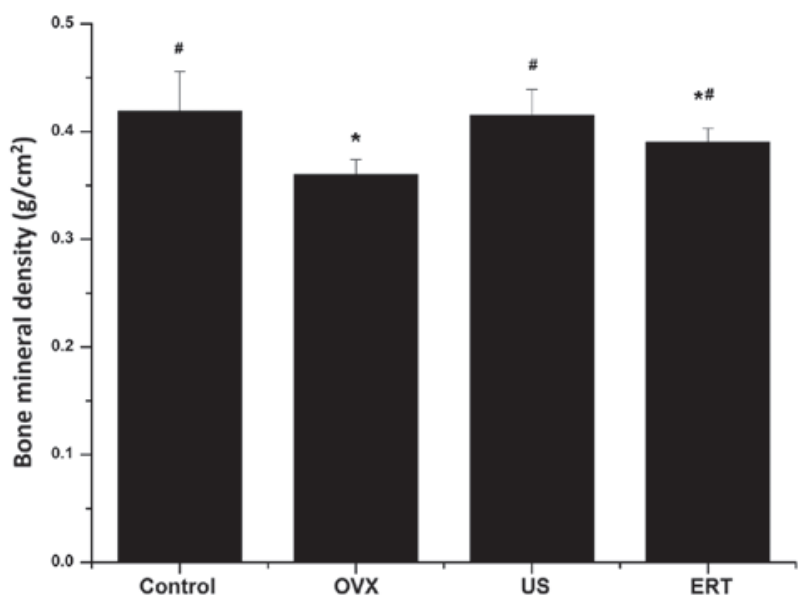

Figure 2. Bone mineral density in the control, OVX, US and ERT groups. ${ }^{*} \mathrm{P}<0.05$ vs. the control group; ${ }^{\prime \prime} \mathrm{P}<0.05$ vs. the OVX group. Values are expressed as the mean \pm standard deviation. OVX, ovariectomized; US, ultrasound; ERT, estrogen replacement therapy.

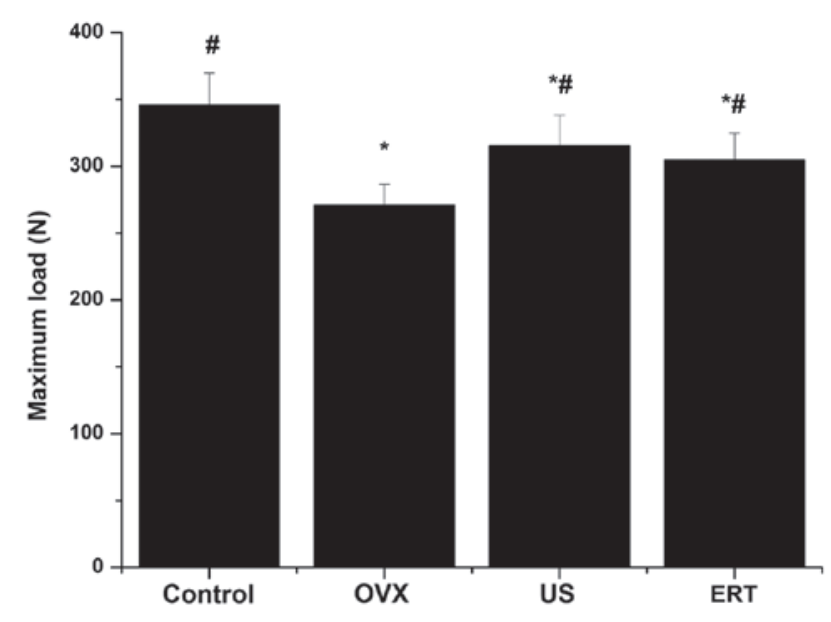

Figure 3. Maximum load value obtained by biomechanical testing in the control, OVX, US and ERT groups. "P<0.05 vs. control group; ${ }^{*} \mathrm{P}<0.05$ vs. OVX group. Values are expressed as the mean \pm standard deviation. OVX, ovariectomized; US, ultrasound; ERT, estrogen replacement therapy.

\section{Results}

Serum estradiol assay. The serum 17ß-estradiol levels among the groups at various time points are shown in Fig. 1 . No statistically significant difference was observed among the $17 \beta$-estradiol levels of the groups prior to ovariectomy at week 0 , indicating that the baseline estradiol levels in all groups were comparable. After the ovariectomy at week 8 , the serum estradiol level was significantly reduced in rabbits that underwent an ovariectomy compared with the control group, indicating that the model of ovariectomy was established successfully. At week 10, at 2 weeeks after the start of the intervention, the levels of serum estradiol in the US and ERT groups were significantly higher compared with those in the OVX group. No statistically significant difference was observed between the 17ß-estradiol levels in the US and ERT groups. Although the estradiol level was increased following the US and ERT treatment of the OVX rabbits, it remained significantly reduced compared with that in the control group (Fig. 1).

$B M D$. As shown in Fig. 2, there were no statistically significant differences in the femur BMD of the US group compared with the control group 2 weeks after the start of treatment; however, the BMD in the ERT group was significantly reduced compared with that in the control group. The US and ERT groups exhibited significantly higher BMD compared with the OVX group $(\mathrm{P}<0.001$ and 0.027 , respectively; Fig. 2).

Bone biomechanical strength testing. The maximum load of the femurs in the OVX, US and ERT groups were statistically reduced compared with the control group, as determined by the three-point bending test; however, the maximum load was significantly higher following the intervention with US and ERT compared with the OVX group (Fig. 3).

Modified Mankin score. In the OVX group, the cartilage surface exhibited erosion and a limited amount of pannus. The chondrocytes were organized irregularly; hypercellularity and clusters were detected in the middle layer of the cartilage. Severe degradation of the ECM and tidemark crossed by blood vessels were observed. The mean modified Mankin score was assessed to be 6.14 . However, markedly smoother surfaces and mild degeneration of chondrocytes and the ECM were observed in the US and ERT groups compared with the OVX group. Furthermore, the mean modified Mankin scores of the US and ERT groups (2.85 and 3.28 points, respectively) were significantly reduced compared with the score of the OVX group, but significantly higher compared with that of the control group. In the control group, healthy cartilage 


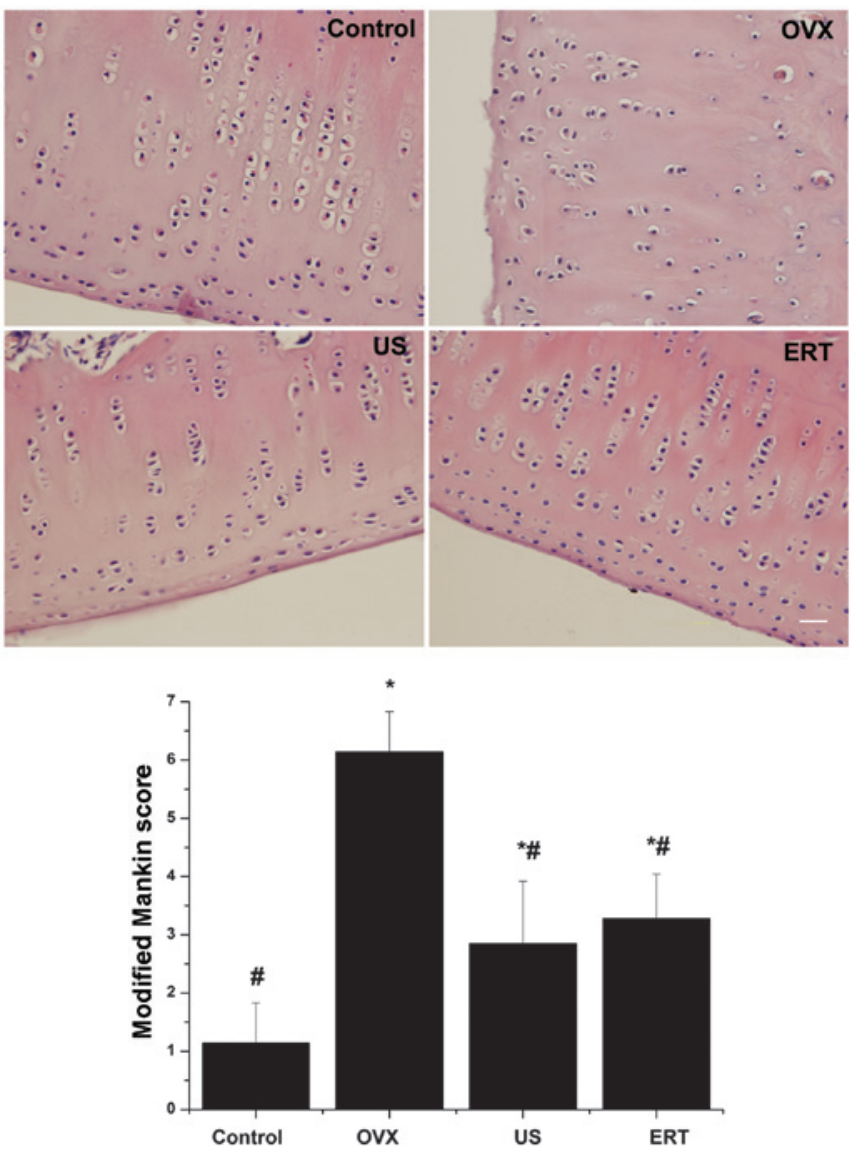

Figure 4. Hematoxylin and eosin-stained cartilage of distal femur from the control, OVX, US and ERT groups (scale bar, $50 \mu \mathrm{m}$ ). Modified Mankin score was calculated for each group. " $\mathrm{P}<0.05$ vs. control group; ${ }^{\text {" }} \mathrm{P}<0.05$ vs. OVX group. Values are expressed as the mean \pm standard deviation. OVX, ovariectomized; US, ultrasound; ERT, estrogen replacement therapy.

with a smooth surface, normal chondrocyte arrangement and ECM were detected. According to the modified Mankin score system, the control group received the lowest score of 1.14 points (Fig. 4).

$R T-q P C R$. The mRNA expression of MMP-13 in the cartilage relative to that of GAPDH was assessed using RT-qPCR. As shown in Fig. 5, the expression levels of GAPDH mRNA exhibited no difference among the four groups. The mRNA expression levels of MMP-13 in the OVX group were markedly higher compared with those in the control group. US treatment led to a significant reduction in MMP-13 mRNA expression compared with the OVX group, but did not statistically differ compared with the control group. The expression levels of MMP-13 mRNA in the ERT group were significantly lower than those in the OVX group, but remained higher compared with those in the control group (Fig. 5).

Western blot analysis. MMP-13 protein expression relative to that of GAPDH was detected by western blot analysis. As shown in Fig. 6, there was no difference in GAPDH expression among the four groups. MMP-13 expression increased notably in the OVX group compared with the control group. MMP-13 protein expression in US group was significantly
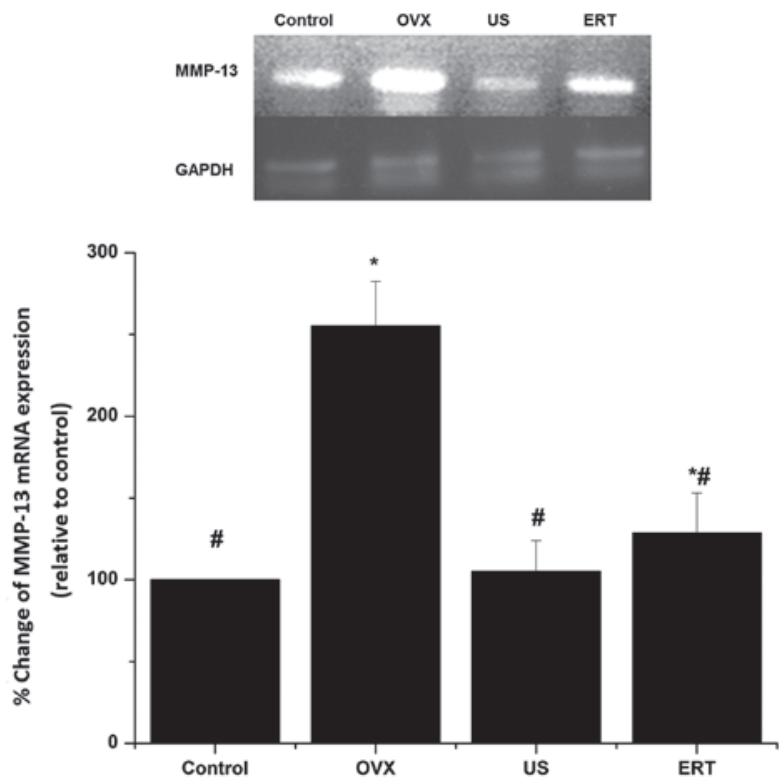

Figure 5. MMP-13 mRNA expression in the control, OVX, US and ERT groups. Each experimental group of MMP-13 mRNA was normalized against GAPDH and expressed as the percentage of control group. "P $<0.05$ vs. control group; ${ }^{\mathrm{P}}<0.05$ vs. OVX group. Values are expressed as the mean \pm standard deviation. OVX, ovariectomized; US, ultrasound; ERT, estrogen replacement therapy; MMP-13, matrix metalloproteinase-13; GAPDH, glyceraldehyde 3-phosphate dehydrogenase.
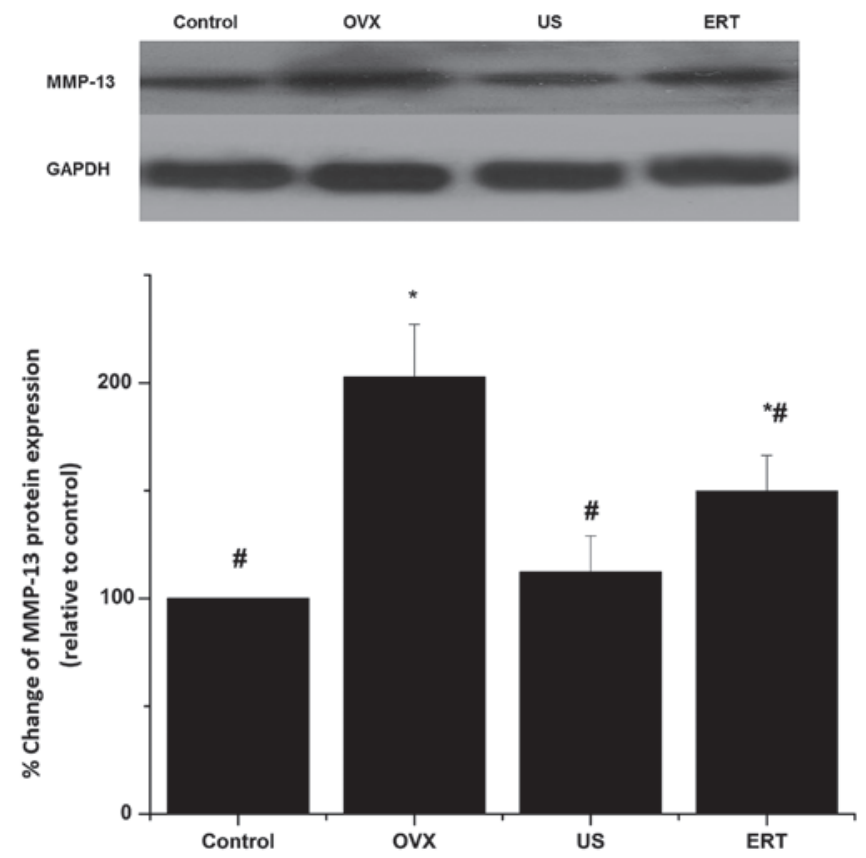

Figure 6. Western blot analysis with antibody specific to MMP-13. Each experimental group of MMP-13 protein was normalized against GAPDH and expressed as the percentage of the control group. ${ }^{*} \mathrm{P}<0.05$ vs. control group; ${ }^{~} \mathrm{P}<0.05$ vs. OVX group. Values are expressed as the mean \pm standard deviation. OVX, ovariectomized; US, ultrasound; ERT, estrogen replacement therapy; MMP-13, matrix metalloproteinase-13; GAPDH, glyceraldehyde 3-phosphate dehydrogenase.

reduced compared with that in the OVX group but did not statistically differ from that in the control group. The expression levels of MMP-13 protein in the ERT group were 
significantly reduced in comparison with those in the OVX group but higher than the control group levels. The differences of MMP-13 protein expression among the four groups were in accordance with the differences in mRNA expression level (Fig. 6).

\section{Discussion}

A marked increase has been observed in the incidence of OA and OP among postmenopausal women (33). The manner in which bone loss occurs in postmenopausal OP is determined by the differences in skeletal metabolism and architecture. Furthermore, estrogen deficiency accelerates cartilage turnover by increasing the surface erosion of the cartilage and the expression of MMP-13 (34), leading to the pathological manifestations associated with OA. In the present study, the OVX and control groups indicate that ovariectomy in rabbits significantly decreased the level of serum estrogen, reduced BMD and bone biomechanical function, eroded the cartilage surface, and increased the mRNA and protein expression levels of MMP-13, indicating that reduced levels of estradiol are associated with the development of OA and OP.

In the present study, the levels of serum estradiol were significantly increased in the US group compared with those in the OVX group, and the effect was comparable to that of estrogen treatment. However, the biological mechanisms underlying the effects of US on the estradiol level remain unclear. It may be hypothesized that US is able to increase estradiol levels in OVX rabbits by regulating the endocrine and immune systems. Thus, further studies are required to investigate the possible signaling pathways involved in the US-mediated regulation of estradiol level.

The results of the present study demonstrate that the BMD and maximum femur load were increased following 2 weeks of US therapy or estrogen treatment. At least 20 million women in developed countries are estimated to be currently receiving hormone replacement therapy (HRT) to prevent postmenopausal OP (35). A meta-analysis based on trials measuring BMD indicated that the use of HRT is associated with a reduction of non-vertebral fractures, in particular in women that receive HRT prior to reaching the age of 60 years (36). Bourrin et al reported that 5 months of treatment with selective estrogen receptor modulator therapy (SERM) increased tibia BMD and strength, but failed to correct cancellous bone architecture (37). However, studies reported as early as 1949 first suggested that US might stimulate osteogenesis (38). The biophysics of US are categorized as thermal or non-thermal effects. The ability of US to stimulate changes in tissues and cells may be due to an increase in temperature and associated energy absorption, which may affect specific enzymes, including MMPs (39). Furthermore, differences observed in tissues and cells following US treatment may be associated with non-thermal processes such as acoustic streaming and caviation (40). A previous study suggested that US therapy may induce an increase in protein synthesis and directly modulate cell membrane permeability, which may increase micromechanical blood pressure leading to accelerated osteogenesis (41). A number of studies have reported an increase in BMD and mechanical strength and stiffness within the initial 7-38 days of US treatment of rabbit tibia (18,42-44). Furthermore, low-intensity ultrasound stimulation in OVX mice is able to activate new bone formation and maintain bone structure, preventing estrogen deficiency-induced bone loss (45). The data presented in the present study suggest that 2 weeks of US and ERT treatment increased the BMD and maximum bone load in OVX rabbits, which is consistent with the results of previous studies.

The results also demonstrated that lesions of the knee joint cartilage were attenuated by the US and estrogen therapy. Estrogen usage in postmenopausal women has been considered as a potential treatment for OA (46). Estrogen exerts its effects on target tissues by binding to the activating estrogen receptor (ER). Estrogen may affect OA formation via MMP expression. Type II collagen is the primary collagen present in articular cartilage, and MMP-13 degrades type II collagen. It has previously been reported that $17 \beta$-estradiol promotes the synthesis of type II collagen, in addition to other proteins such as insulin in articular chondrocytes (47). Lee et al demonstrated that $17 \beta$-estradiol inhibits the expression of MMP-13 in chondrocytes (48). Lu et al reported that estrogen deficiency may result in increased expression of MMP-13 (49). US has been applied in a number of in vitro and in vivo studies to certify its ability to increase the self-repair capacity of cartilage with OA (17-20). Molecular studies have shown increased chondrocyte differentiation as a result of increased aggrecan expression, proteoglycan synthesis and upregulation of chondroitin sulfate release following US treatment (23). The results of the present study demonstrate improved cartilage structure and lost cellular matrix straining following US treatment. Furthermore, the results of the RT-qPCR and western blot analysis indicated an overall reduction in the transcription and translation of MMP-13, indicating that US is able to attenuate type II collagen digestion by reducing the transcription and translation of MMP-13. Similar results were obtained by Gurkan et al, who demonstrated that MMP-13 expression was decreased as a result of US stimulation (50). In addition, Naito et al reported that low-intensity pulsed ultrasound (LIPUS) increased the articular cartilage type II collagen in a rat osteoarthritis model (51). However, a negative result was obtained by Park et al, who observed unchanged MMP-13 mRNA levels following LIPUS treatment (52). In the present study, US and estrogen therapy appeared to mitigate cartilage degradation by inhibiting the transcription and translation of MMP-13.

Although ERT may be applied to preserve bone structure and articular cartilage structural integrity in OVX rabbits, a major drawback of long-term ERT is an increased risk of breast cancer (53), endometrial cancer (54) and ovarian cancer (55). US therapy has fewer side-effects, and there is evidence that US therapy has a positive effect on bone and cartilage regeneration $(18,20,23)$. US treatment may be applicable to the treatment of women with postmenopausal OA and OP. Whether different intensities, frequencies and treatment times of US result in different effects on serum estrogen level, BMD, bone biomechanical strength and MMP-13 expression requires further investigated. Further studies are also required to determine the molecular mechanisms underlying the effects of US treatment on postmenopausal OA and OP.

In conclusion, the results of the present study demonstrate that within 8 weeks of undergoing ovariectomy, rabbits exhibited reductions in serum estradiol levels, BMD and 
bone biomechanical strength, and increased transcription and expression of MMP-13, which may be associated with alterations in articular cartilage structure. Following 2 weeks of US and estrogen therapy post-ovariectomy, the serum estradiol levels had reduced, BMD and bone biomechanical function were protected, and the degradation of articular cartilage in OVX rabbits had slowed. These effects may have been mediated by the downregulation of MMP-13 gene expression and translation. Furthermore, by comparing the responses of bone and cartilage to US and estradiol therapy, it is concluded that US was superior to ERT as a therapy as fewer side-effects are caused by US treatment.

\section{Acknowledgements}

The authors thank Dr Cao Xueqing for her assistance with the hematoxylin and eosin staining.

\section{References}

1. Abramson SB and Attur M: Developments in the scientific understanding of osteoarthritis. Arthritis Res Ther 11: 227, 2009.

2. Tetlow LC, Adlam DJ and Woolley DE: Matrix metalloproteinase and proinflammatory cytokine production by chondrocytes of human osteoarthritic cartilage: Associations with degenerative changes. Arthritis Rheum 44: 585-594, 2001.

3. Knäuper V, López-Otin C, Smith B, Knight G and Murphy G: Biochemical characterization of human collagenase-3. J Biol Chem 271: 1544-1550, 1996.

4. Poole AR, Kobayashi M, Yasuda T, Laverty S, Mwale F, Kojima T, Sakai T, Wahl C, El-Maadawy S, Webb G, et al: Type II collagen degradation and its regulation in articular cartilage in osteoarthritis. Ann Rheum Dis 61 (Suppl 2): ii78-ii81, 2002.

5. Sun SS, Ma HL, Liu CL, Huang CH, Cheng CK and Wei HW: Difference in femoral head and neck material properties between osteoarthritis and osteoporosis. Clin Biomech (Bristol, Avon) 23 (Suppl 1): S39-S47, 2008.

6. Cooley HM, Stankovich J and Jones G: The association between hormonal and reproductive factors and hand osteoarthritis. Maturitas 45: 257-265, 2003.

7. Wluka AE, Cicuttini FM and Spector TD: Menopause, oestrogens and arthritis. Maturitas 35: 183-199, 2000.

8. Ivey JL and Baylink DJ: Postmenopausal osteoporosis : Proposed roles of defective coupling and estrogen deficiency. Metab Bone Dis Relat Res 3: 3-7, 1981.

9. Høegh-Andersen P, TankóLB, Andersen TL, Lundberg CV, Mo JA, Heegaard AM, Delaissé JM and Christgau S: Ovariectomized rats as a model of postmenopausal osteoarthritis: Validation and application. Arthritis Res Ther 6: R169-R180, 2004.

10. Dai G, Wang S, Li J, Liu C and Liu Q: The validity of osteoarthritis model induced by bilateral ovariectomy in guinea pig. J Huazhong Univ Sci Technolog Med Sci 26: 716-719, 2006.

11. Turner R, Maran A, Lotinun S, Hefferan T, Evans GL, Zhang M and Sibonga JD: Animal models for osteoporosis. Rev Endocr Metab Disord 2: 117-127, 2001.

12. Kalu DN: The ovariectomized rat model of postmenopausal bone loss. Bone Miner 15: 175-191, 1991.

13. Roman-Blas JA, Castañeda S, Largo R and Herrero-Beaumont G: Osteoarthritis associated with estrogen deficiency. Arthritis Res Ther 11: 241, 2009.

14. Grady D, Rubin SM, Petitti DB, Fox CS, Black D, Ettinger B Ernster VL and Cummings SR: Hormone therapy to prevent disease and prolong life in postmenopausal women. Ann Intern Med 117: 1016-1037, 1992.

15. Gallagher JC and Sai AJ: Molecular biology of bone remodeling: Implications for new therapeutic targets for osteoporosis Maturitas 65: 301-307, 2010.

16. Baker KG, Robertson VJ and Duck FA: A review of therapeutic ultrasound: Biophysical effects. Phys Ther 81: 1351-1358, 2001

17. Tsumaki N, Kakiuchi M, Sasaki J, Ochi T and Yoshikawa H: Low-intensity pulsed ultrasound accelerates maturation of callus in patients treated with opening-wedge high tibial osteotomy by hemicallotasis. J Bone Joint Surg Am 86-A: 2399-2405, 2004.
18. Shimazaki A, Inui K, Azuma Y, Nishimura N and Yamano Y: Low-intensity pulsed ultrasound accelerates bone maturation in distraction osteogenesis in rabbits. J Bone Joint Surg Br 82: 1077-1082, 2000

19. Cook SD, Salkeld SL, Patron LP, Doughty ES and Jones DG: The effect of low-intensity pulsed ultrasound on autologous osteochondral plugs in a canine model. Am J Sports Med 36: 1733-1741, 2008.

20. Perry MJ, Parry LK, Burton VJ, Gheduzzi S, Beresford JN, Humphrey VF and Skerry TM: Ultrasound mimics the effect of mechanical loading on bone formation in vivo on rat ulnae. Med Eng Phys 31: 42-47, 2009.

21. Unsworth J, Kaneez S, Harris S, Ridgway J, Fenwick S, Chenery D and Harrison A: Pulsed low intensity ultrasound enhances mineralisation in preosteoblast cells. Ultrasound Med Biol 33: 1468-1474, 2007.

22. Nishikori T, Ochi M,Uchio Y,Maniwa S, Kataoka H, KawasakiK, Katsube K and Kuriwaka M: Effects of low-intensity pulsed ultrasound on proliferation and chondroitin sulfate synthesis of cultured chondrocytes embedded in Atelocollagen gel. J Biomed Mater Res 59: 201-206, 2002

23. Parvizi J, Wu CC, Lewallen DG, Greenleaf JF and Bolander ME: Low-intensity ultrasound stimulates proteoglycan synthesis in rat chondrocytes by increasing aggrecan gene expression. J Orthop Res 17: 488-494, 1999.

24. Loyola-Sánchez A, Richardson J and MacIntyre NJ: Efficacy of ultrasound therapy for the management of knee osteoarthritis: A systematic review with meta-analysis. Osteoarthritis Cartilage 18: 1117-1126, 2010.

25. Warden SJ, Bennell KL, Forwood MR, McMeeken JM and Wark JD: Skeletal effects of low-intensity pulsed ultrasound on the ovariectomized rodent. Ultrasound Med Biol 27: 989-998, 2001.

26. Castañeda S, Largo R, Calvo E, Rodríguez-Salvanés F, Marcos ME, Díaz-Curiel M and Herrero-Beaumont G: Bone mineral measurements of subchondral and trabecular bone in healthy and osteoporotic rabbits. Skeletal Radiol 35: 34-41, 2006.

27. Zeng D, Luo Q, Lin H, Zhang J and He C: The effect of therapeutic ultrasound to apoptosis of chondrocyte and caspase- 3 and caspase- 8 expression in rabbit surgery-induced model of knee osteoarthritis. Rheumatol Int 32: 3771-3777, 2012.

28. Chen J, Huang LQ, Xia QJ and He C-Q: Effects of pulsed electromagnetic fields on the mRNA expression of CAII and RANK in ovariectomized rats. Rheumatol Int 32: 1527-1532, 2012.

29. Li S, Luo Q, Huang L, Hu Y, Xia Q and He C: Effects of pulsed electromagnetic fields on cartilage apoptosis signalling pathways in ovariectomised rats. Int Orthop 35: 1875-1882, 2011.

30. Mazess R, Collick B, Trempe J, Barden H and Hanson J: Performance evaluation of a dual energy $\mathrm{x}$-ray bone densitometer. Calcif Tissue Int 44: 228, 1989.

31. Cohen B and Rushton N: Accuracy of DEXA measurement of bone mineral density after total hip arthroplasty. J Bone Joint Surg Br 77: 479, 1995

32. Mankin H, Dorfman H, Lippiello L and Zarins A: Biochemical and metabolic abnormalities in articular cartilage from osteo-arthritic human hips. II. Correlation of morphology with biochemical and metabolic data. J Bone Joint Surg Am 53: 523-537, 1971.

33. Kanis JA: Estrogens, the menopause and osteoporosis. Bone 19 (Suppl 5): 185S-190S, 1996.

34. Lu T, Achari Y, Rattner JB and Hart DA: Evidence that estrogen receptor enhances MMP-13 promoter activity in HIG-82 cells and that this enhancement can be influenced by ligands and involves specific promoter sites. Biochem Cell Biol 85: 326-336, 2007.

35. Dören M: Estrogen therapy for prevention and treatment of osteoporosis. Maturitas 43 (Suppl): S53-S56, 2002.

36. Torgerson DJ and Bell-Syer SE: Hormone replacement therapy and prevention of nonvertebral fractures: A meta-analysis of randomized trials. JAMA 285: 2891-2897, 2001.

37. Bourrin S, Ammann P, Bonjour JP and Rizzoli R: Recovery of proximal tibia bone mineral density and strength, but not cancellous bone architecture, after long-term bisphosphonate or selective estrogen receptor modulator therapy in aged rats. Bone 30: 195-200, 2002.

38. Buchtala V: Present state of ultrasound therapy. Dia Med 22: 2944-2950, 1950.

39. Claes L and Willie B: The enhancement of bone regeneration by ultrasound. Prog Biophys Mol Biol 93: 384-398, 2007.

40. Chang WH, Sun JS, Chang SP and Lin JC: Study of thermal effects of ultrasound stimulation on fracture healing. Bioelectromagnetics 23: 256-263, 2002. 
41. Dyson M: Non-thermal cellular effects of ultrasound. Br J Cancer Suppl: 165-171, 1982.

42. Sakurakichi K, Tsuchiya H, Uehara K, Yamashiro T, Tomita K and Azuma Y: Effects of timing of low-intensity pulsed ultrasound on distraction osteogenesis. J Orthop Res 22: 395-403, 2004.

43. Uglow MG, Peat RA, Hile MS, Bilston LE, Smith EJ and Little DG: Low-intensity ultrasound stimulation in distraction osteogenesis in rabbits. Clin Orthop Relat Res 303-312, 2003.

44. Tis JE, Meffert RH, Inoue N, McCarthy EF, Machen MS McHale KA and Chao EY: The effect of low intensity pulsed ultrasound applied to rabbit tibiae during the consolidation phase of distraction osteogenesis. J Orthop Res 20: 793-800, 2002.

45. Lim D, Ko CY, Seo DH, Woo DG, Kim JM, Chun KJ and Kim HS: Low-intensity ultrasound stimulation prevents osteoporotic bone loss in young adult ovariectomized mice. J Orthop Res 29: 116-125, 2011.

46. Yang KGA, Saris DBF, Dhert WJA and Verbout AJ: Osteoarthritis of the knee: Current treatment options and future directions. Curr Orthop 18: 311-320, 2004.

47. Claassen H, Schluter M, Schunke M and Kurz B: Influence of 17beta-estradiol and insulin on type II collagen and protein synthesis of articular chondrocytes. Bone 39: 310-317, 2006.

48. Lee YJ, Lee EB, Kwon YJ, Lee JJ, Cho WS, Kim HA and Song YW: Effect of estrogen on the expression of matrix metalloproteinase (MMP)-1, MMP-3 and MMP-13 and tissue inhibitor of metalloproternase-1 in osteoarthritis chondrocytes. Rheumatol Int 23: 282-288, 2003.
49. Lu T, Achari Y, Sciore P and Hart DA: Estrogen receptor alpha regulates matrix metalloproteinase-13 promoter activity primarily through the AP-1 transcriptional regulatory site. Biochim Biophys Acta 1762: 719-731, 2006.

50. Gurkan I, Ranganathan A, Yang X, Horton WE Jr, Todman M, Huckle J, Pleshko N and Spencer RG: Modification of osteoarthritis in the guinea pig with pulsed low-intensity ultrasound treatment. Osteoarthritis Cartilage 18: 724-733, 2010.

51. Naito K, Watari T, Muta T, Furuhata A, Iwase H, Igarashi M, Kurosawa H, Nagaoka I and Kaneko K: Low-intensity pulsed ultrasound (LIPUS) increases the articular cartilage type II collagen in a rat osteoarthritis model. J Orthop Res 28: 361-369, 2009.

52. Park K, Hoffmeister B, Han DK and Hasty K: Therapeutic ultrasound effects on interleukin-1beta stimulated cartilage construct in vitro. Ultrasound Med Biol 33: 286-295, 2007.

53. Collaborative Group on Hormonal Factors in Breast Cancer: Breast cancer and hormone replacement therapy: Collaborative reanalysis of data from 51 epidemiological studies of 52,705 women with breast cancer and 108,411 women without breast cancer. Lancet 350: 1047-1059, 1997.

54. Grady D, Gebretsadik T, Kerlikowske K, Ernster V and Petitti D Hormone replacement therapy and endometrial cancer risk: A meta-analysis. Obstet Gynecol 85: 304-313, 1995.

55. Rodriguez C, Patel AV, Calle EE, Jacob EJ and Thun MJ: Estrogen replacement therapy and ovarian cancer mortality in a large prospective study of US women. JAMA 285: 1460-1465, 2001 . 